Kragujevac Journal of Mathematics

Volume 44(3) (2020), Pages 443-458.

\title{
CONVERGENCE OF DOUBLE COSINE SERIES
}

\author{
KARANVIR SINGH ${ }^{1}$ AND KANAK MODI $^{2}$
}

\begin{abstract}
In this paper we consider double cosine series whose coefficients form a null sequence of bounded variation of order $(p, 0),(0, p)$ and $(p, p)$ with the weight $(j k)^{p-1}$ for some $p>1$. We study pointwise convergence, uniform convergence and convergence in $L^{r}$-norm of the series under consideration. In a certain sense our results extend the results of Young [7], Kolmogorov [3] and Móricz [4,5].
\end{abstract}

\section{INTRODUCTION}

Consider the double cosine series

$$
\sum_{j=0}^{\infty} \sum_{k=0}^{\infty} \lambda_{j} \lambda_{k} a_{j k} \cos j x \cos k y,
$$

on positive quadrant $T=[0, \pi] \times[0, \pi]$ of the two dimensional torus where $\lambda_{0}=\frac{1}{2}$ and $\lambda_{j}=1$ for $j=1,2,3, \ldots$.

The rectangular partial sums $S_{m n}(x, y)$ and the Cesàro means $\sigma_{m n}(x, y)$ of the series (1.1) are defined as

$$
\begin{aligned}
& S_{m n}(x, y)=\sum_{j=0}^{m} \sum_{k=0}^{n} \lambda_{j} \lambda_{k} a_{j k} \cos j x \cos k y \\
& \sigma_{m n}(x, y)=\frac{1}{(m+1)(n+1)} \sum_{j=0}^{m} \sum_{k=0}^{n} S_{j k}(x, y), \quad m, n>0
\end{aligned}
$$

Key words and phrases. Rectangular partial sums, $L^{r}$-convergence, Cesàro means, monotone sequences.

2010 Mathematics Subject Classification. Primary: 42A20, 42A32.

DOI 10.46793/KgJMat2003.443S

Received: September 17, 2017.

Accepted: June 05, 2018. 
and for $\lambda>1$, the truncated Cesáro means are defined by

$$
V_{m n}^{\lambda}(x, y)=\frac{1}{([\lambda m]-m)([\lambda n]-n)} \sum_{j=m+1}^{[\lambda m]} \sum_{k=n+1}^{[\lambda n]} S_{j k}(x, y) .
$$

Now assuming the coefficients $\left\{a_{j k}: j, k \geq 0\right\}$ in (1.1) be a double sequence of real numbers which satisfy the following conditions for some positive integer $p$ :

$$
\begin{gathered}
\left|a_{j k}\right|(j k)^{p-1} \rightarrow 0 \text { as } \max \{j, k\} \rightarrow \infty, \\
\lim _{k \rightarrow \infty} \sum_{j=0}^{\infty}\left|\triangle_{p 0} a_{j k}\right|(j k)^{p-1}=0, \\
\lim _{j \rightarrow \infty} \sum_{k=0}^{\infty}\left|\triangle_{0 p} a_{j k}\right|(j k)^{p-1}=0, \\
\sum_{j=0}^{\infty} \sum_{k=0}^{\infty}\left|\triangle_{p p} a_{j k}\right|(j k)^{p-1}<\infty .
\end{gathered}
$$

The finite order differences $\triangle_{p q} a_{j k}$ are defined by

$$
\begin{aligned}
& \triangle_{00} a_{j k}=a_{j k}, \\
& \triangle_{p q} a_{j k}=\triangle_{p-1, q} a_{j k}-\triangle_{p-1, q} a_{j+1, k}, \quad p \geq 1, q \geq 0, \\
& \triangle_{p q} a_{j k}=\triangle_{p, q-1} a_{j k}-\triangle_{p, q-1} a_{j, k+1}, \quad p \geq 0, q \geq 1 .
\end{aligned}
$$

Also a double induction argument gives

$$
\triangle_{p q} a_{j k}=\sum_{s=0}^{p} \sum_{t=0}^{q}(-1)^{s+t}\left(\begin{array}{l}
p \\
s
\end{array}\right)\left(\begin{array}{l}
q \\
t
\end{array}\right) a_{j+s, k+t} .
$$

We can call the above mentioned conditions (1.2)-(1.5) as conditions of bounded variation of order $(p, 0),(0, p)$ and $(p, p)$ respectively with the weight $(j k)^{p-1}$. Obviously these conditions generalise the concept of monotone sequences. Also any sequence satisfying (1.5) with $p=2$ is called a quasi-convex sequence $[3,5]$. Clearly the conditions (1.3) and (1.4) can be derived from (1.2) and (1.5) for $p=1$ and moreover for $p=1$, the conditions (1.2) and (1.5) reduce to $\left|a_{j k}\right| \rightarrow 0$ as $\max \{j, k\} \rightarrow \infty$ and

$$
\sum_{j=0}^{\infty} \sum_{k=0}^{\infty}\left|\triangle_{11} a_{j k}\right|<\infty \text {. }
$$

Generally the pointwise convergence of the series (1.1) is defined in Pringsheim's sense ([8], Vol. 2, Ch. 17) which means that the rectangular partial sums of the type

$$
S_{m n}(x, y)=\sum_{j=0}^{m} \sum_{k=0}^{n} \lambda_{j} \lambda_{k} a_{j k} \cos j x \cos k y, \quad m, n \geq 0,
$$

are formed and then by taking both $m, n$ tend to $\infty$ (independently of one another) the limit $f(x, y)$ (provided it exists) is assigned to the series (1.1) as its sum. 
Also let $\|f\|_{r}$ denotes the $L^{r}\left(T^{2}\right)$-norm, i.e,

$$
\|f\|_{r}=\left(\int_{0}^{\pi} \int_{0}^{\pi}|f(x, y)|^{r} d x d y\right)^{1 / r}, \quad 1 \leq r<\infty
$$

and $\|f\|$ denotes $L^{1}\left(T^{2}\right)$-norm, i.e,

$$
\|f\|=\int_{0}^{\pi} \int_{0}^{\pi}|f(x, y)| d x d y
$$

In this paper, we will investigate the validity of the following statements:

(a) $S_{m n}(x, y)$ converges pointwise to $f(x, y)$ for every $(x, y) \in T^{2}$;

(b) $S_{m n}(x, y)$ converges uniformly to $f(x, y)$ on $T^{2}$;

(c) $\left\|S_{m n}(x, y)-f(x, y)\right\|_{r}=o(1)$ as $\min \{m, n\} \rightarrow 0$.

Such type of problems have been studied by Young [7] and Kolmogorov [3] for onedimensional case (single trigonometric series especially cosine series ) and by Móricz [4, 5] and K. Kaur, Bhatia and Ram [2] for double trigonometric series. In [5], Móricz studied both double cosine series and double sine series as far as their integrability and convergence in $L^{1}$-norm is concerned where as in [4] he studied double trigonometric series of the form

$$
\sum_{-\infty}^{\infty} \sum_{-\infty}^{\infty} c_{j k} e^{i(j x+k y)}
$$

under coefficients of bounded variation. All of them discussed the case for $p=1$ or $p=2$ only. Our aim in this paper is to extend the above results from $p=1$ to general cases for double cosine series.

In the results, $C_{p}$ and $C_{p r}$ denote constants which may not be the same at each occurrence. Also we write $\lambda_{n}=[\lambda n]$ where $\mathrm{n}$ is a positive integer, $\lambda>1$ is a real number and $[\cdot]$ means greatest integral part.

The first main result reads as follows.

Theorem 1.1. Assume that conditions (1.2)-(1.5) are satisfied for some $p \geq 1$, then

(i) $S_{m n}(x, y)$ converges pointwise to $f(x, y)$ for every $(x, y) \in T^{2}$ such that $x, y>0$;

(ii) $\left\|S_{m n}(x, y)-f(x, y)\right\|_{r}=o(1)$ as $\min \{m, n\} \rightarrow \infty, 1 \leq r<\infty$.

The above theorem has been proved by Móricz [4,5] for $p=1$ and $p=2$ using suitable estimates for Dirichlet's kernel $D_{j}(x)$ and Fejér kernel $K_{j}(x)$. In the case of a single series for $p=2$, the results regarding convergence have been proved by Kolmogorov [3].

Obviously, condition (1.5) implies any of the following conditions:

$$
\lim _{\lambda \downarrow 1} \varlimsup_{n \rightarrow \infty} \sum_{j=0}^{\infty} \sum_{k=n+1}^{\lambda_{n}} \frac{\lambda_{n}-k+1}{\lambda_{n}-n}\left|\triangle_{p p} a_{j k}\right|(j k)^{p-1}=0,
$$




$$
\lim _{\lambda \downarrow 1} \varlimsup_{m \rightarrow \infty} \sum_{j=m+1}^{\lambda_{m}} \sum_{k=0}^{\infty} \frac{\lambda_{m}-j+1}{\lambda_{m}-m}\left|\triangle_{p p} a_{j k}\right|(j k)^{p-1}=0 .
$$

We introduce the following three sums for $m, n \geq 0$ and $\lambda>1$ :

$$
\begin{aligned}
& \sum_{10}^{\lambda}(m, n, x, y)=\sum_{j=m+1}^{\lambda_{m}} \sum_{k=0}^{n} \frac{\lambda_{m}-j+1}{\lambda_{m}-m} a_{j k} \cos j x \cos k y, \\
& \sum_{01}^{\lambda}(m, n, x, y)=\sum_{j=0}^{m} \sum_{k=n+1}^{\lambda_{n}} \frac{\lambda_{n}-k+1}{\lambda_{n}-n} a_{j k} \cos j x \cos k y, \\
& \sum_{11}^{\lambda}(m, n, x, y)=\sum_{j=m+1}^{\lambda_{m}} \sum_{k=n+1}^{\lambda_{n}} \frac{\lambda_{m}-j+1}{\lambda_{m}-m} \frac{\lambda_{n}-k+1}{\lambda_{n}-n} a_{j k} \cos j x \cos k y
\end{aligned}
$$

and we have

$$
\begin{aligned}
& \sum_{11}^{\lambda}(m, n ; x, y)=\frac{1}{\left(\lambda_{m}-m\right)} \sum_{u=m+1}^{\lambda_{m}}\left(\sum_{01}^{\lambda}(u, n ; x, y)-\sum_{01}^{\lambda}(m, n ; x, y)\right) \\
& \sum_{11}^{\lambda}(m, n ; x, y)=\frac{1}{\left(\lambda_{n}-n\right)} \sum_{v=n+1}^{\lambda_{n}}\left(\sum_{10}^{\lambda}(m, v ; x, y)-\sum_{10}^{\lambda}(m, n ; x, y)\right) .
\end{aligned}
$$

This implies

$$
\sum_{11}^{\lambda}(m, n ; x, y) \leq\left\{\begin{array}{c}
2 \sup _{m \leq u \leq \lambda_{m}}\left(\left|\sum_{01}^{\lambda}(u, n ; x, y)\right|\right) \\
2 \sup _{n \leq v \leq \lambda_{n}}\left(\left|\sum_{10}^{\lambda}(m, v ; x, y)\right|\right)
\end{array}\right\}
$$

The second result of this paper is the following theorem.

Theorem 1.2. $\quad$ (i) Let $E \subset T^{2}$. Assume that the following conditions are satisfied:

$$
\begin{aligned}
& \lim _{\lambda \downarrow 1} \varlimsup_{m, n \rightarrow \infty}\left(\sup _{(x, y) \in E}\left|\sum_{10}^{\lambda}(m, n ; x, y)\right|\right)=0, \\
& \lim _{\lambda \downarrow 1} \varlimsup_{m, n \rightarrow \infty}\left(\sup _{(x, y) \in E}\left|\sum_{01}^{\lambda}(m, n ; x, y)\right|\right)=0 .
\end{aligned}
$$

If $V_{m n}^{\lambda}(x, y)$ converges uniformly to $f(x, y)$ on $E \subset T^{2}$ as $\min \{m, n\} \rightarrow \infty$ (that is, in the unrestricted sense), then so does $S_{m n}$.

(ii) Assume that the following conditions are satisfied for some $r \geq 1$ :

$$
\begin{aligned}
& \lim _{\lambda \downarrow 1} \varlimsup_{m, n \rightarrow \infty}\left(\left\|\sum_{10}^{\lambda}(m, n ; x, y)\right\|_{r}\right)=0, \\
& \lim _{\lambda \downarrow 1} \varlimsup_{m, n \rightarrow \infty}\left(\left\|\sum_{01}^{\lambda}(m, n ; x, y)\right\|_{r}\right)=0 .
\end{aligned}
$$

If $\left\|V_{m n}^{\lambda}-f\right\|_{r} \rightarrow 0$ unrestictedly then $\left\|S_{m n}-f\right\|_{r} \rightarrow 0$ as $\min \{m, n\} \rightarrow \infty$.

We will also prove the following theorem. 
Theorem 1.3. Assume that the conditions (1.2)-(1.4) and (1.6)-(1.7) are satisfied for some $p \geq 1$, then

(i) if $V_{m n}^{\lambda}(x, y)$ converges uniformly to $f(x, y)$ as $\min \{m, n\} \rightarrow \infty$, then so does $S_{m n}$

(ii) if $\left\|V_{m n}^{\lambda}-f\right\|_{r} \longrightarrow 0$ unrestictedly for some $r$ with $1 \leq r<\infty$, then $\left\|S_{m n}-f\right\|_{r} \longrightarrow 0$ as $\min \{m, n\} \rightarrow \infty$.

\section{Notation And Formulas}

We define for every $\alpha=0,1,2, \ldots$ the sequence $S_{0}^{\alpha}, S_{1}^{\alpha}, S_{2}^{\alpha}, \ldots$ by the conditions

$$
S_{n}^{0}=S_{n}, \quad S_{n}^{\alpha}=\sum_{u=0}^{n} S_{u}^{\alpha-1}, \quad \alpha \geq 1
$$

and

$$
A_{n}^{0}=1, \quad A_{n}^{\alpha}=\sum_{u=0}^{n} A_{u}^{\alpha-1}, \quad \alpha \geq 1,
$$

denotes binomial coefficients. Also

$$
A_{n}^{\alpha}=\left(\begin{array}{c}
n+\alpha \\
n
\end{array}\right) \simeq \frac{n^{\alpha}}{\Gamma(\alpha+1)}, \quad \alpha \neq-1,-2,-3, \ldots
$$

The Cesàro means $T_{n}^{\alpha}$ of order $\alpha$ of $\sum a_{n}$ will be defined by $T_{n}^{\alpha}=\frac{S_{n}^{\alpha}}{A_{n}^{\alpha}}$ and also it is known [8] that $\int_{0}^{\pi}\left|T_{n}^{\alpha}(x)\right| d x, \alpha>0$, is bounded for all $n$.

\section{LEMmaS}

We require the following lemmas for the proof of our results.

Lemma 3.1. For $m, n \geq 0$ and $p>1$, the following representation holds:

$$
\begin{aligned}
S_{m n}(x, y)= & \sum_{j=0}^{m} \sum_{k=0}^{n} \lambda_{j} \lambda_{k} a_{j k} \cos j x \cos k y \\
= & \sum_{j=0}^{m} \sum_{k=0}^{n} \triangle_{p p} a_{j k} S_{j}^{p-1}(x) S_{k}^{p-1}(y)+\sum_{j=0}^{m} \sum_{t=0}^{p-1} \triangle_{p t} a_{j, n+1} S_{j}^{p-1}(x) S_{n}^{t}(y) \\
& +\sum_{k=0}^{n} \sum_{s=0}^{p-1} \triangle_{s p} a_{m+1, k} S_{m}^{s}(x) S_{k}^{p-1}(y)+\sum_{s=0}^{p-1} \sum_{t=0}^{p-1} \triangle_{s t} a_{m+1, n+1} S_{m}^{s}(x) S_{n}^{t}(y) .
\end{aligned}
$$

Lemma $3.2([1])$. For $m, n \geq 0$ and $\lambda>1$, the following representation holds:

$$
\begin{aligned}
S_{m n}-\sigma_{m n}= & \frac{\lambda_{m}+1}{\lambda_{m}-m} \frac{\lambda_{n}+1}{\lambda_{n}-n}\left(\sigma_{\lambda_{m}, \lambda_{n}}-\sigma_{\lambda_{m}, n}-\sigma_{m, \lambda_{n}}+\sigma_{m n}\right) \\
& +\frac{\lambda_{m}+1}{\lambda_{m}-m}\left(\sigma_{\lambda_{m}, n}-\sigma_{m n}\right)+\frac{\lambda_{n}+1}{\lambda_{n}-n}\left(\sigma_{m, \lambda_{n}}-\sigma_{m n}\right) \\
& -\sum_{11}^{\lambda}(m, n, x, y)-\sum_{10}^{\lambda}(m, n, x, y)-\sum_{01}^{\lambda}(m, n, x, y) .
\end{aligned}
$$


Lemma 3.3. For $m, n \geq 0$ and $\lambda>1$, we have the following representation:

$$
V_{m n}^{\lambda}-S_{m n}=\sum_{11}^{\lambda}(m, n, x, y)+\sum_{10}^{\lambda}(m, n, x, y)+\sum_{01}^{\lambda}(m, n, x, y) .
$$

Proof. We have

$$
V_{m n}^{\lambda}(x, y)=\frac{1}{\left(\lambda_{m}-m\right)\left(\lambda_{n}-n\right)} \sum_{j=m+1}^{\lambda_{m}} \sum_{k=n+1}^{\lambda_{n}} S_{j k}(x, y) .
$$

Performing double summation by parts, we have

$$
\begin{aligned}
V_{m n}^{\lambda}= & \frac{\lambda_{m}+1}{\lambda_{m}-m} \frac{\lambda_{n}+1}{\lambda_{n}-n} \sigma_{\lambda_{m}, \lambda_{n}}-\frac{\lambda_{m}+1}{\lambda_{m}-m} \frac{n+1}{\lambda_{n}-n} \sigma_{\lambda_{m}, n} \\
& -\frac{m+1}{\lambda_{m}-m} \frac{\lambda_{n}+1}{\lambda_{n}-n} \sigma_{m, \lambda_{n}}+\frac{m+1}{\lambda_{m}-m} \frac{n+1}{\lambda_{n}-n} \sigma_{m n} \\
= & \frac{\lambda_{m}+1}{\lambda_{m}-m} \frac{\lambda_{n}+1}{\lambda_{n}-n}\left(\sigma_{\lambda_{m}, \lambda_{n}}-\sigma_{\lambda_{m}, n}-\sigma_{m, \lambda_{n}}+\sigma_{m n}\right) \\
& +\frac{\lambda_{m}+1}{\lambda_{m}-m}\left(\sigma_{\lambda_{m}, n}-\sigma_{m n}\right)+\frac{\lambda_{n}+1}{\lambda_{n}-n}\left(\sigma_{m, \lambda_{n}}-\sigma_{m n}\right)+\sigma_{m n} .
\end{aligned}
$$

The use of Lemma 3.2, gives

$$
\begin{aligned}
V_{m n}^{\lambda}-S_{m n}= & \sum_{j=m+1}^{\lambda_{m}} \sum_{k=n+1}^{\lambda_{n}} \frac{\lambda_{m}-j+1}{\lambda_{m}-m} \frac{\lambda_{n}-k+1}{\lambda_{n}-n} a_{j k} \cos j x \cos k y \\
& +\sum_{j=m+1}^{\lambda_{m}} \sum_{k=0}^{n} \frac{\lambda_{m}-j+1}{\lambda_{m}-m} a_{j k} \cos j x \cos k y \\
& +\sum_{j=0}^{m} \sum_{k=n+1}^{\lambda_{n}} \frac{\lambda_{n}-k+1}{\lambda_{n}-n} a_{j k} \cos j x \cos k y .
\end{aligned}
$$

Lemma 3.4. For $m, n \geq 0$ and $\lambda>1$, we have the following representation:

$$
\begin{aligned}
\sum_{10}^{\lambda}(m, n ; x, y)= & \sum_{j=m+1}^{\lambda_{m}} \sum_{k=0}^{n} \frac{\lambda_{m}-j+1}{\lambda_{m}-m} a_{j k} \cos j x \cos k y \\
= & \sum_{j=m+1}^{\lambda_{m}} \sum_{k=0}^{n} \frac{\lambda_{m}-j+1}{\lambda_{m}-m} \triangle_{p p} a_{j k} S_{j}^{p-1}(x) S_{k}^{p-1}(y) \\
& +\sum_{j=m+1}^{\lambda_{m}} \sum_{t=0}^{p-1} \frac{\lambda_{m}-j+1}{\lambda_{m}-m} \triangle_{p t} a_{j, n+1} S_{j}^{p-1}(x) S_{n}^{t}(y) \\
& +\frac{1}{\lambda_{m}-m} \sum_{j=m+1}^{\lambda_{m}} \sum_{s=0}^{p-1} \sum_{k=0}^{n} \triangle_{s p} a_{j+1, k} S_{j}^{s}(x) S_{k}^{p-1}(y) \\
& +\frac{1}{\lambda_{m}-m} \sum_{j=m+1}^{\lambda_{m}} \sum_{s=0}^{p-1} \sum_{t=0}^{p-1} \triangle_{s t} a_{j+1, n+1} S_{j}^{s}(x) S_{n}^{t}(y)
\end{aligned}
$$




$$
\begin{aligned}
& -\sum_{s=0}^{p-1} \sum_{k=0}^{n} \triangle_{s p} a_{m+1, k} S_{m}^{s}(x) S_{k}^{p-1}(y) \\
& -\sum_{s=0}^{p-1} \sum_{t=0}^{p-1} \triangle_{s t} a_{m+1, n+1} S_{m}^{s}(x) S_{n}^{t}(y) .
\end{aligned}
$$

Proof. We have by summation by parts,

$$
\begin{aligned}
& \sum_{10}^{\lambda}(m, n ; x, y) \\
& =\sum_{k=0}^{n} \cos k y\left(\sum_{j=m+1}^{\lambda_{m}} \frac{\lambda_{m}-j+1}{\lambda_{m}-m} a_{j k} \cos j x\right) \\
& =\sum_{k=0}^{n} \cos k y\left(\sum_{j=m+1}^{\lambda_{m}} \frac{\lambda_{m}-j+1}{\lambda_{m}-m} \triangle_{p 0} a_{j k} S_{j}^{p-1}(x)\right. \\
& \left.+\frac{1}{\lambda_{m}-m} \sum_{j=m+1}^{\lambda_{m}} \sum_{s=0}^{p-1} \triangle_{s 0} a_{j+1, k} S_{j}^{s}(x)-\sum_{s=0}^{p-1} \triangle_{s 0} a_{m+1, k} S_{m}^{s}(x)\right) \\
& =\sum_{j=m+1}^{\lambda_{m}} \frac{\lambda_{m}-j+1}{\lambda_{m}-m} S_{j}^{p-1}(x)\left(\sum_{k=0}^{n} \triangle_{p 0} a_{j k} \cos k y\right) \\
& +\frac{1}{\lambda_{m}-m} \sum_{j=m+1}^{\lambda_{m}} \sum_{s=0}^{p-1}\left(\sum_{k=0}^{n} \triangle_{s 0} a_{j+1, k} \cos k y\right) S_{j}^{s}(x) \\
& -\sum_{s=0}^{p-1}\left(\sum_{k=0}^{n} \triangle_{s 0} a_{m+1, k} \cos k y\right) S_{m}^{s}(x) \\
& =\sum_{j=m+1}^{\lambda_{m}} \frac{\lambda_{m}-j+1}{\lambda_{m}-m} S_{j}^{p-1}(x)\left(\sum_{k=0}^{n} \triangle_{p p} a_{j k} S_{k}^{p-1}(y)+\sum_{t=0}^{p-1} \triangle_{p t} a_{j, n+1} S_{n}^{t}(y)\right) \\
& +\frac{1}{\lambda_{m}-m} \sum_{j=m+1}^{\lambda_{m}} \sum_{s=0}^{p-1}\left(\sum_{k=0}^{n} \triangle_{s p} a_{j+1, k} S_{k}^{p-1}(y)+\sum_{t=0}^{p-1} \triangle_{s t} a_{j+1, n+1} S_{n}^{t}(y)\right) S_{j}^{s}(x) \\
& -\sum_{s=0}^{p-1}\left(\sum_{k=0}^{n} \triangle_{s p} a_{m+1, k} S_{k}^{p-1}(y)+\sum_{t=0}^{p-1} \triangle_{s t} a_{m+1, n+1} S_{n}^{t}(y)\right) S_{m}^{s}(x) .
\end{aligned}
$$

Similarly we can have representation for $\sum_{01}^{\lambda}(m, n ; x, y)$.

\section{Proof of Theorems}

Proof of Theorem 1.1. For $m, n \geq 0$ and $p>1$, we have from Lemma 3.1,

$$
\begin{aligned}
S_{m n}(x, y)= & \sum_{j=0}^{m} \sum_{k=0}^{n} \triangle_{p p} a_{j k} S_{j}^{p-1}(x) S_{k}^{p-1}(y)+\sum_{j=0}^{m} \sum_{t=0}^{p-1} \triangle_{p t} a_{j, n+1} S_{j}^{p-1}(x) S_{n}^{t}(y) \\
& +\sum_{k=0}^{n} \sum_{s=0}^{p-1} \triangle_{s p} a_{m+1, k} S_{m}^{s}(x) S_{k}^{p-1}(y)+\sum_{s=0}^{p-1} \sum_{t=0}^{p-1} \triangle_{s t} a_{m+1, n+1} S_{m}^{s}(x) S_{n}^{t}(y)
\end{aligned}
$$




$$
=\sum_{1}+\sum_{2}+\sum_{3}+\sum_{4}
$$

Using the results as given in $[6]$ that $S_{j}^{p}(x)=O\left(\frac{1}{x^{p}}\right)$, for all $p \geq 2,0<x \leq \pi$, etc, we have for $0<x, y \leq \pi$,

$$
\sum_{j=0}^{\infty} \sum_{k=0}^{\infty}\left|\triangle_{p p} a_{j k} S_{j}^{p-1}(x) S_{k}^{p-1}(y)\right|<\infty \quad(\text { by }(1.2))
$$

and also by (1.3)-(1.5), we have

$$
\begin{aligned}
\sum_{j=0}^{m} \sum_{t=0}^{p-1} \triangle_{p t} a_{j, n+1} & \leq \sum_{t=0}^{p-1} \sum_{v=0}^{t}\left(\begin{array}{l}
t \\
v
\end{array}\right)\left(\sum_{j=0}^{m}\left|\triangle_{p 0} a_{j, n+v+1}\right|\right) \\
& \leq \sup _{n<k \leq n+p} \sum_{j=0}^{m}\left|\triangle_{p 0} a_{j k}\right| \\
& \leq \sup _{n<k \leq n+p} \sum_{j=0}^{m}\left|\triangle_{p 0} a_{j k}\right| \rightarrow 0 \text { as } \min \{m, n\} \rightarrow \infty .
\end{aligned}
$$

Thus,

$$
\sum_{j=0}^{m} \sum_{t=0}^{p-1} \triangle_{p t} a_{j, n+1} S_{j}^{p-1}(x) S_{n}^{t}(y) \rightarrow 0 \text { as } \min \{m, n\} \rightarrow \infty .
$$

And similarly

$$
\begin{aligned}
\sum_{s=0}^{p-1} \sum_{k=0}^{n} \triangle_{s p} a_{m+1, k} & \leq \sum_{s=0}^{p-1} \sum_{u=0}^{s}\left(\begin{array}{l}
s \\
u
\end{array}\right)\left(\sum_{k=0}^{n}\left|\triangle_{0 p} a_{m+u+1, k}\right|\right) \\
& \leq \sup _{m<j \leq m+p} \sum_{k=0}^{n}\left|\triangle_{0 p} a_{j k}\right| \\
& \leq \sup _{m<j \leq m+p} \sum_{k=0}^{n}\left|\triangle_{0 p} a_{j k}\right| \rightarrow 0 \text { as } \min \{m, n\} \rightarrow \infty .
\end{aligned}
$$

Thus,

$$
\sum_{k=0}^{n} \sum_{s=0}^{p-1} \triangle_{s p} a_{m+1, k} S_{m}^{s}(x) S_{k}^{p-1}(y) \rightarrow 0,
$$

as $\min \{m, n\} \rightarrow \infty$. Also

$$
\begin{aligned}
\sum_{s=0}^{p-1} \sum_{t=0}^{p-1} \triangle_{s t} a_{m+1, n+1} & \leq \sum_{s=0}^{p-1} \sum_{t=0}^{p-1} \sum_{u=0}^{s} \sum_{v=0}^{t}\left(\begin{array}{l}
s \\
u
\end{array}\right)\left(\begin{array}{l}
t \\
v
\end{array}\right)\left|\triangle_{00} a_{m+u+1, n+v+1}\right| \\
& \leq \sup _{j>m, k>n}\left|a_{j k}\right| \rightarrow 0 \text { as } \min \{m, n\} \rightarrow \infty .
\end{aligned}
$$

So,

$$
\sum_{s=0}^{p-1} \sum_{t=0}^{p-1} \triangle_{s t} a_{m+1, n+1} S_{m}^{s}(x) S_{n}^{t}(y) \rightarrow 0 \text { as } \min \{m, n\} \rightarrow \infty .
$$


Consequently, series (1.1) converges to the function $f(x, y)$ where

$$
f(x, y)=\sum_{j=0}^{\infty} \sum_{k=0}^{\infty} \triangle_{p p} a_{j k} S_{j}^{p-1}(x) S_{k}^{p-1}(y) \quad \text { and } \quad \lim _{m, n \rightarrow \infty} S_{m n}(x, y)=f(x, y) .
$$

Now we will calculate $\left\|\sum_{1}\right\|,\left\|\sum_{2}\right\|,\left\|\sum_{3}\right\|$ and $\left\|\sum_{4}\right\|$ in the following way:

$$
\begin{aligned}
& \left\|\sum_{1}\right\|=\left\|\sum_{j=0}^{m} \sum_{k=0}^{n} \triangle_{p p} a_{j k} S_{j}^{p-1}(x) S_{k}^{p-1}(y)\right\| \\
& \leq \sum_{j=0}^{m} \sum_{k=0}^{n}\left|\triangle_{p p} a_{j k}\right| \int_{0}^{\pi} \int_{0}^{\pi}\left|S_{j}^{p-1}(x) S_{k}^{p-1}(y)\right| d x d y \\
& \leq \sum_{j=0}^{m} \sum_{k=0}^{n}\left|\triangle_{p p} a_{j k}\right| A_{j}^{p-1} A_{k}^{p-1} \int_{0}^{\pi} \int_{0}^{\pi}\left|T_{j}^{p-1}(x) T_{k}^{p-1}(y)\right| d x d y \\
& \leq C_{p} \sum_{j=0}^{m} \sum_{k=0}^{n}\left|\triangle_{p p} a_{j k}\right| j^{p-1} k^{p-1}, \\
& \left\|\sum_{2}\right\|=\left\|\sum_{j=0}^{m} \sum_{t=0}^{p-1} \triangle_{p t} a_{j, n+1} S_{j}^{p-1}(x) S_{n}^{t}(y)\right\| \\
& \leq \sum_{t=0}^{p-1} \sum_{v=0}^{t}\left(\begin{array}{l}
t \\
v
\end{array}\right)\left(\sum_{j=0}^{m}\left|\triangle_{p 0} a_{j, n+v+1}\right|\right) A_{j}^{p-1} A_{n}^{t} \int_{-\pi}^{\pi} \int_{-\pi}^{\pi}\left|T_{j}^{p-1}(x) T_{n}^{t}(y)\right| d x d y \\
& \leq C_{p} \sup _{n<k \leq n+p} \sum_{j=0}^{m}\left|\triangle_{p 0} a_{j k}\right| j^{p-1}\left(\sum_{t=0}^{p-1} n^{t}\right) \\
& \leq C_{p} \sup _{n<k \leq n+p} \sum_{j=0}^{m}\left|\triangle_{p 0} a_{j k}\right| j^{p-1} k^{p-1}, \\
& \left\|\sum_{3}\right\|=\left\|\sum_{s=0}^{p-1} \sum_{k=0}^{n} \triangle_{s p} a_{m+1, k} S_{m}^{s}(x) S_{k}^{p-1}(y)\right\| \\
& \leq \sum_{s=0}^{p-1} \sum_{u=0}^{s}\left(\begin{array}{l}
s \\
u
\end{array}\right)\left(\sum_{k=0}^{n}\left|\triangle_{0 p} a_{m+u+1, k}\right|\right) A_{m}^{s} A_{k}^{p-1} \int_{0}^{\pi} \int_{0}^{\pi}\left|T_{m}^{s}(x) T_{k}^{p-1}(y)\right| d x d y \\
& \leq C_{p} \sup _{m<j \leq m+p} \sum_{k=0}^{n}\left|\triangle_{0 p} a_{j k}\right| k^{p-1}\left(\sum_{s=0}^{p-1} m^{s}\right) \\
& \leq C_{p} \sup _{m<j \leq m+p} \sum_{k=0}^{n}\left|\triangle_{0 p} a_{j k}\right| j^{p-1} k^{p-1}, \\
& \left\|\sum_{4}\right\|=\left\|\sum_{s=0}^{p-1} \sum_{t=0}^{p-1} \triangle_{s t} a_{m+1, n+1} S_{m}^{s}(x) S_{n}^{t}(y)\right\|
\end{aligned}
$$




$$
\begin{aligned}
& \leq \sum_{s=0}^{p-1} \sum_{t=0}^{p-1} \sum_{u=0}^{s} \sum_{v=0}^{t}\left(\begin{array}{l}
s \\
u
\end{array}\right)\left(\begin{array}{l}
t \\
v
\end{array}\right)\left|\triangle_{00} a_{m+u+1, n+v+1}\right| A_{m}^{s} A_{n}^{t} \int_{0}^{\pi} \int_{0}^{\pi}\left|T_{m}^{s}(x) T_{n}^{t}(y)\right| d x d y \\
& \leq C_{p} \sup _{j>m, k>n}\left|a_{j k}\right| j^{p-1} k^{p-1} .
\end{aligned}
$$

Now let $R_{m n}$ consists of all $(j, k)$ with $j>m$ or $k>n$, that is,

$$
\sum \sum_{(j, k) \in R_{m n}}=\sum_{j=m+1}^{\infty} \sum_{k=0}^{n}+\sum_{j=0}^{\infty} \sum_{k=n+1}^{\infty}+\sum_{j=m+1}^{\infty} \sum_{k=n+1}^{\infty} .
$$

Then

$$
\begin{aligned}
\left\|f-S_{m n}\right\|_{r}= & \left(\int_{0}^{\pi} \int_{0}^{\pi}\left|f(x, y)-S_{m n}(x, y)\right|^{r} d x d y\right)^{1 / r}, 1 \leq r<\infty \\
\leq & \left\|\sum_{(j, k) \in R_{m n}} \triangle_{p p} a_{j k} S_{j}^{p-1}(x) S_{k}^{p-1}(y)\right\|_{r} \\
& +\left\|\sum_{j=0}^{m} \sum_{t=0}^{p-1} \triangle_{p t} a_{j, n+1} S_{j}^{p-1}(x) S_{n}^{t}(y)\right\|_{r} \\
& +\left\|\sum_{k=0}^{n} \sum_{s=0}^{p-1} \triangle_{s p} a_{m+1, k} S_{m}^{s}(x) S_{k}^{p-1}(y)\right\|_{r} \\
& +\left\|\sum_{s=0}^{p-1} \sum_{t=0}^{p-1} \triangle_{s t} a_{m+1, n+1} S_{m}^{s}(x) S_{n}^{t}(y)\right\|_{r} \\
\leq & C_{p r}\left\{\left(\sum_{(j, k) \in R_{m n}}\left|\triangle_{p p} a_{j k}\right| j^{p-1} k^{p-1}\right)\right. \\
& +\left(\sup _{n<k \leq n+p} \sum_{j=0}^{m}\left|\triangle_{p 0} a_{j k}\right| j^{p-1} k^{p-1}\right) \\
& +\left(\sup _{m<j \leq m+p} \sum_{k=0}^{n}\left|\triangle_{0 p} a_{j k}\right| j^{p-1} k^{p-1}\right) \\
& \left.\left.+\left(\sup _{j>m, k>n}\left|a_{j k}\right| j^{p-1} k^{p-1}\right)\right\} \quad \text { as discussed above }\right) \\
\min _{k m}\{m\} \rightarrow \infty & (\text { by }(1.2)-(1.5)),
\end{aligned}
$$

which proves (ii) part.

Proof of Theorem 1.2. Using the relation (1.8), we find that (1.9) or (1.10) implies

$$
\lim _{\lambda \downarrow 1} \varlimsup_{m, n \rightarrow \infty}\left(\sup _{(x, y) \in E}\left|\sum_{11}^{\lambda}(m, n ; x, y)\right|\right)=0 .
$$


Assume that $V_{m n}^{\lambda}(x, y)$ converges uniformly on E to $f(x, y)$. Then by Lemma 3.3, we get

$$
\begin{aligned}
\varlimsup_{m, n \rightarrow \infty}\left(\left|\sup _{(x, y) \in E}\left(S_{m n}(x, y)-V_{m n}^{\lambda}(x, y)\right)\right|\right) \leq & \varlimsup_{m, n \rightarrow \infty}\left(\sup _{(x, y) \in E}\left|\sum_{10}^{\lambda}(m, n ; x, y)\right|\right) \\
& +\varlimsup_{m, n \rightarrow \infty}\left(\sup _{(x, y) \in E}\left|\sum_{01}^{\lambda}(m, n ; x, y)\right|\right) \\
& +\varlimsup_{m, n \rightarrow \infty}\left(\sup _{(x, y) \in E}\left|\sum_{11}^{\lambda}(m, n ; x, y)\right|\right) .
\end{aligned}
$$

After taking $\lambda \downarrow 1$ the result follows from (1.9), (1.10) and (4.1).

For (ii) part of theorem, we have

$$
\begin{aligned}
\left\|\sum_{11}^{\lambda}(m, n ; x, y)\right\|_{r} & =\frac{1}{\lambda_{m}-m} \sum_{u=m+1}^{\lambda_{m}}\left(\left\|\sum_{01}^{\lambda}(u, n ; x, y)\right\|_{r}+\left\|\sum_{01}^{\lambda}(m, n ; x, y)\right\|_{r}\right) \\
& \leq 2\left(\sup _{m \leq u \leq \lambda_{m}}\left(\left\|\sum_{01}^{\lambda}(u, n ; x, y)\right\|_{r}\right)\right) .
\end{aligned}
$$

Thus (1.11) implies

$$
\lim _{\lambda \downarrow 1} \varlimsup_{m, n \rightarrow \infty}\left\|\sum_{11}^{\lambda}(m, n ; x, y)\right\|_{r}=0 .
$$

Thus, the result of Theorem 1.2 (ii) follows.

Proof of Theorem 1.3. Using the Lemma 3.4, we can write the expression for $\sum_{01}^{\lambda}(m, n ; x, y)$ as

$$
\begin{aligned}
\sum_{01}^{\lambda}(m, n ; x, y)= & \sum_{j=0}^{m} \sum_{k=n+1}^{\lambda_{n}} \frac{\lambda_{n}-k+1}{\lambda_{n}-n} a_{j k} \cos j x \cos k y \\
= & \sum_{j=0}^{m} \sum_{k=n+1}^{\lambda_{n}} \frac{\lambda_{n}-k+1}{\lambda_{n}-n} \triangle_{p p} a_{j k} S_{j}^{p-1}(x) S_{k}^{p-1}(y) \\
& +\sum_{k=n+1}^{\lambda_{n}} \sum_{s=0}^{p-1} \frac{\lambda_{n}-k+1}{\lambda_{n}-n} \triangle_{s p} a_{m+1, k} S_{m}^{s}(x) S_{k}^{p-1}(y) \\
& +\frac{1}{\lambda_{n}-n} \sum_{j=0}^{m} \sum_{k=n+1}^{\lambda_{n}} \sum_{t=0}^{p-1} \triangle_{p t} a_{j, k+1} S_{j}^{p-1}(x) S_{k}^{t}(y) \\
& +\frac{1}{\lambda_{n}-n} \sum_{k=n+1}^{\lambda_{n}} \sum_{s=0}^{p-1} \sum_{t=0}^{p-1} \triangle_{s t} a_{m+1, k+1} S_{m}^{s}(x) S_{k}^{t}(y) \\
& -\sum_{t=0}^{p-1} \sum_{j=0}^{m} \triangle_{p t} a_{j, n+1} S_{j}^{p-1}(x) S_{n}^{t}(y)
\end{aligned}
$$




$$
\begin{aligned}
& -\sum_{s=0}^{p-1} \sum_{t=0}^{p-1} \triangle_{s t} a_{m+1, n+1} S_{m}^{s}(x) S_{n}^{t}(y) \\
= & I_{1}+I_{2}+I_{3}+I_{4}+I_{5}+I_{6} .
\end{aligned}
$$

Now by using (1.2)-(1.4) and (1.6) along with estimates of $S_{j}^{p-1}(x)$ etc., as mentioned in [6], we have the following estimates in brief:

$$
\begin{aligned}
\left|I_{1}\right| & =\left|\sum_{j=0}^{m} \sum_{k=n+1}^{\lambda_{n}} \frac{\lambda_{n}-k+1}{\lambda_{n}-n} \triangle_{p p} a_{j k} S_{j}^{p-1}(x) S_{k}^{p-1}(y)\right| \\
& \leq \sum_{j=0}^{m} \sum_{k=n+1}^{\lambda_{n}} \frac{\lambda_{n}-k+1}{\lambda_{n}-n}\left|\triangle_{p p} a_{j k}\right| \\
& \rightarrow 0 \quad \text { as } \min \{m, n\} \rightarrow \infty .
\end{aligned}
$$

Consequently, $\lim _{\lambda \downarrow 1} \varlimsup_{m, n \rightarrow \infty}\left(\sup _{(x, y) \in E}\left|I_{1}\right|\right) \rightarrow 0$ as $\min \{m, n\} \rightarrow \infty$. Also,

$$
\begin{aligned}
\left|I_{2}\right| & =\left|\sum_{k=n+1}^{\lambda_{n}} \sum_{s=0}^{p-1} \frac{\lambda_{n}-k+1}{\lambda_{n}-n} \triangle_{s p} a_{m+1, k} S_{m}^{s}(x) S_{k}^{p-1}(y)\right| \\
& \leq \sum_{s=0}^{p-1} \sum_{u=0}^{s}\left(\begin{array}{l}
s \\
u
\end{array}\right) \sum_{k=n+1}^{\lambda_{n}}\left|\triangle_{0 p} a_{m+u+1, k}\right| \\
& \leq \sup _{m<j \leq m+p} \sum_{k=n+1}^{\lambda_{n}}\left|\triangle_{0 p} a_{j k}\right| \rightarrow 0 \quad \text { as } \min \{m, n\} \rightarrow \infty .
\end{aligned}
$$

So, $\lim _{\lambda \downarrow 1} \varlimsup_{m, n \rightarrow \infty}\left(\sup _{(x, y) \in E}\left|I_{2}\right|\right) \rightarrow 0$ as $\min \{m, n\} \rightarrow \infty$. Also,

$$
\begin{aligned}
\left|I_{3}\right| & \leq \sup _{n<k \leq \lambda_{n}} \sum_{t=0}^{p-1} \sum_{j=0}^{m}\left|\triangle_{p t} a_{j, k+1}\right| \\
& \leq \sup _{n<k \leq \lambda_{n}} \sum_{t=0}^{p-1} \sum_{v=0}^{t}\left(\begin{array}{l}
t \\
v
\end{array}\right) \sum_{j=0}^{m}\left|\triangle_{p t} a_{j, k+v+1}\right| \\
& \leq \sup _{n<k \leq \lambda_{n}+p} \sum_{j=0}^{m}\left|\triangle_{p 0} a_{j k}\right| \rightarrow 0 \text { as } \min \{m, n\} \rightarrow \infty
\end{aligned}
$$

which implies $\lim _{\lambda \downarrow 1} \varlimsup_{m, n \rightarrow \infty}\left(\sup _{(x, y) \in E}\left|I_{3}\right|\right) \rightarrow 0$ as $\min \{m, n\} \rightarrow \infty$. Now,

$$
\begin{aligned}
\left|I_{4}\right| & \leq \sup _{n<k \leq \lambda_{n}} \sum_{s=0}^{p-1} \sum_{t=0}^{p-1}\left|\triangle_{s t} a_{m+1, k+1}\right| \\
& \leq \sup _{j>m, k>n}\left|a_{j k}\right| \rightarrow 0 \text { as } \min \{m, n\} \rightarrow \infty
\end{aligned}
$$


Thus $\lim _{\lambda \downarrow 1} \varlimsup_{m, n \rightarrow \infty}\left(\sup _{(x, y) \in E}\left|I_{4}\right|\right) \rightarrow 0$ as $\min \{m, n\} \rightarrow \infty$. Also,

$$
\left|I_{5}\right| \leq \sum_{t=0}^{p-1} \sum_{v=0}^{t}\left(\begin{array}{l}
t \\
v
\end{array}\right) \sum_{j=0}^{m}\left|\triangle_{p 0} a_{j, n+v+1}\right| \leq \sup _{n<k \leq n+p} \sum_{j=0}^{m}\left|\triangle_{p 0} a_{j k}\right| \rightarrow 0 \text { as } \min \{m, n\} \rightarrow \infty,
$$

which implies $\lim _{\lambda \downarrow 1} \varlimsup_{m, n \rightarrow \infty}\left(\sup _{(x, y) \in E}\left|I_{5}\right|\right) \rightarrow 0$ as $\min \{m, n\} \rightarrow \infty$. Also,

$$
\begin{aligned}
\left|I_{6}\right| & \leq \sum_{s=0}^{p-1} \sum_{t=0}^{p-1} \sum_{u=0}^{s} \sum_{v=0}^{t}\left(\begin{array}{l}
s \\
u
\end{array}\right)\left(\begin{array}{l}
t \\
v
\end{array}\right)\left|\triangle_{00} a_{m+u+1, n+v+1}\right| \\
& \leq \sup _{j>m, k>n}\left|a_{j k}\right| \rightarrow 0 \text { as } \min \{m, n\} \rightarrow \infty,
\end{aligned}
$$

and

$$
\lim _{\lambda \downarrow 1} \varlimsup_{m, n \rightarrow \infty}\left(\sup _{(x, y) \in E}\left|I_{6}\right|\right) \rightarrow 0 \text { as } \min \{m, n\} \rightarrow \infty .
$$

Thus, combining all these, we have

$$
\lim _{\lambda \downarrow 1} \varlimsup_{m, n \rightarrow \infty}\left(\sup _{(x, y) \in E}\left|\sum_{01}^{\lambda}(m, n ; x, y)\right|\right)=0 .
$$

Similarly (1.2)-(1.4) and (1.7) results in

$$
\lim _{\lambda \downarrow 1} \varlimsup_{m, n \rightarrow \infty}\left(\sup _{(x, y) \in E}\left|\sum_{10}^{\lambda}(m, n ; x, y)\right|\right)=0 .
$$

Thus, first part of theorem follows from Theorem 1.2.

Proof of (ii). We have

$$
\left\|S_{m n}-f\right\|_{r} \leq\left\|S_{m n}-V_{m n}^{\lambda}\right\|_{r}+\left\|V_{m n}^{\lambda}-f\right\|_{r} .
$$

By assumption $\left\|V_{m n}^{\lambda}-f\right\|_{r} \rightarrow 0$, so it is sufficient to show that

$$
\left\|S_{m n}-V_{m n}^{\lambda}\right\|_{r} \rightarrow 0 \text { as } \min \{m, n\} \rightarrow \infty .
$$

By Lemma 3.3, we have

$$
\begin{aligned}
\left\|S_{m n}-V_{m n}^{\lambda}\right\|_{r} \leq & \left\|\sum_{10}^{\lambda}(m, n ; x, y)\right\|_{r}+\left\|\sum_{01}^{\lambda}(m, n ; x, y)\right\|_{r} \\
& +\left\|\sum_{11}^{\lambda}(m, n ; x, y)\right\|_{r} .
\end{aligned}
$$

Now in order to estimate $\left\|\sum_{01}^{\lambda}(m, n ; x, y)\right\|_{r}$, we first find $\left\|I_{1}\right\|,\left\|I_{2}\right\|,\left\|I_{3}\right\|,\left\|I_{4}\right\|,\left\|I_{5}\right\|$ and $\left\|I_{6}\right\|$, so we have

$$
\begin{aligned}
\left\|I_{1}\right\| & =\left\|\sum_{j=0}^{m} \sum_{k=n+1}^{\lambda_{n}} \frac{\lambda_{n}-k+1}{\lambda_{n}-n} \triangle_{p p} a_{j k} S_{j}^{p-1}(x) S_{k}^{p-1}(y)\right\| \\
& \leq \sum_{j=0}^{m} \sum_{k=n+1}^{\lambda_{n}} \frac{\lambda_{n}-k+1}{\lambda_{n}-n} \triangle_{p p} a_{j k} A_{j}^{p-1} A_{k}^{p-1} \int_{0}^{\pi} \int_{0}^{\pi}\left|T_{j}^{p-1}(x) T_{k}^{p-1}(y)\right| d x d y
\end{aligned}
$$




$$
\begin{aligned}
& \leq C_{p} \sum_{j=0}^{m} \sum_{k=n+1}^{\lambda_{n}} \frac{\lambda_{n}-k+1}{\lambda_{n}-n}\left|\triangle_{p p} a_{j k}\right| j^{p-1} k^{p-1}, \\
& \left\|I_{2}\right\|=\left\|\sum_{k=n+1}^{\lambda_{n}} \sum_{s=0}^{p-1} \frac{\lambda_{n}-k+1}{\lambda_{n}-n} \triangle_{s p} a_{m+1, k} S_{m}^{s}(x) S_{k}^{p-1}(y)\right\| \\
& \leq C_{p} \sum_{s=0}^{p-1} \sum_{u=0}^{s}\left(\begin{array}{l}
s \\
u
\end{array}\right) \sum_{k=n+1}^{\lambda_{n}}\left|\triangle_{0 p} a_{m+u+1, k}\right| k^{p-1} m^{s} \\
& \leq C_{p} \sup _{m<j \leq m+p}\left(\sum_{k=n+1}^{\lambda_{n}}\left|\triangle_{0 p} a_{j k}\right| k^{p-1}\right)\left(\sum_{s=0}^{p-1} m^{s}\right) \\
& \leq C_{p} \sup _{m<j \leq m+p} \sum_{k=n+1}^{\lambda_{n}}\left|\triangle_{0 p} a_{j k}\right| j^{p-1} k^{p-1}, \\
& \left\|I_{3}\right\| \leq C_{p} \sup _{n<k \leq \lambda_{n}} \sum_{t=0}^{p-1} \sum_{j=0}^{m}\left|\triangle_{p t} a_{j, k+1}\right| j^{p-1} k^{t} \\
& \leq C_{p} \sup _{n<k \leq \lambda_{n}} \sum_{t=0}^{p-1} \sum_{v=0}^{t}\left(\begin{array}{l}
t \\
v
\end{array}\right) \sum_{j=0}^{m}\left|\triangle_{p t} a_{j, k+v+1}\right| j^{p-1} k^{t} \\
& \leq C_{p} \sup _{n<k \leq \lambda_{n}+p} \sum_{j=0}^{m}\left|\triangle_{p 0} a_{j k}\right| j^{p-1} k^{p-1}, \\
& \left\|I_{4}\right\| \leq C_{p} \sup _{n<k \leq \lambda_{n}} \sum_{s=0}^{p-1} \sum_{t=0}^{p-1}\left|\triangle_{s t} a_{m+1, k+1}\right| m^{s} k^{t} \\
& \leq C_{p} \sup _{j>m, k>n}\left|a_{j k}\right| j^{p-1} k^{p-1} \\
& \left\|I_{5}\right\| \leq C_{p} \sum_{t=0}^{p-1} \sum_{v=0}^{t}\left(\begin{array}{l}
t \\
v
\end{array}\right) \sum_{j=0}^{m}\left|\triangle_{p 0} a_{j, n+v+1}\right| j^{p-1} n^{t} \\
& \leq C_{p} \sup _{n<k \leq n+p} \sum_{j=0}^{m}\left|\triangle_{p 0} a_{j k}\right| j^{p-1} k^{p-1}, \\
& \left\|I_{6}\right\| \leq C_{p} \sum_{s=0}^{p-1} \sum_{t=0}^{p-1} \sum_{u=0}^{s} \sum_{v=0}^{t}\left(\begin{array}{l}
s \\
u
\end{array}\right)\left(\begin{array}{l}
t \\
v
\end{array}\right)\left|\triangle_{00} a_{m+u+1, n+v+1}\right| m^{s} n^{t} \\
& \leq C_{p} \sup _{j>m, k>n}\left|a_{j k}\right| j^{p-1} k^{p-1} \text {. }
\end{aligned}
$$

Thus, we can estimate

$$
\begin{aligned}
\left\|\sum_{01}^{\lambda}(m, n ; x, y)\right\|_{r} \leq & C_{p r} \sum_{k=n+1}^{\lambda_{n}} \sum_{j=0}^{m} \frac{\lambda_{n}-k+1}{\lambda_{n}-n}\left|\triangle_{p p} a_{j k}\right| j^{p-1} k^{p-1} \\
& +C_{p r}\left(\sup _{m<j \leq m+p} \sum_{k=n+1}^{\lambda_{n}}\left|\triangle_{0 p} a_{j k}\right| j^{p-1} k^{p-1}\right)
\end{aligned}
$$




$$
\begin{aligned}
& +C_{p r}\left(\sup _{n<k \leq \lambda_{n}+p} \sum_{j=0}^{m}\left|\triangle_{p 0} a_{j k}\right| j^{p-1} k^{p-1}\right) \\
& +C_{p r}\left(\sup _{j>m, k>n}\left|a_{j k}\right| j^{p-1} k^{p-1}\right) \\
& +C_{p r}\left(\sup _{n<k \leq n+p} \sum_{j=0}^{m}\left|\triangle_{p 0} a_{j k}\right| j^{p-1} k^{p-1}\right) \\
& +C_{p r}\left(\sup _{j>m, k>n}\left|a_{j k}\right| j^{p-1} k^{p-1}\right) .
\end{aligned}
$$

By (1.2)-(1.4) and (1.6), we conclude that

$$
\lim _{\lambda \downarrow 1} \varlimsup_{m, n \rightarrow \infty}\left(\left\|\sum_{01}^{\lambda}(m, n ; x, y)\right\|_{r}\right)=0 .
$$

Similarly, by conditions (1.2)-(1.4) and (1.7), we get

$$
\lim _{\lambda \downarrow 1} \varlimsup_{m, n \rightarrow \infty}\left(\left\|\sum_{10}^{\lambda}(m, n ; x, y)\right\|_{r}\right)=0 .
$$

Also, by (1.8), we have

$$
\lim _{\lambda \downarrow 1} \varlimsup_{m, n \rightarrow \infty}\left(\left\|\sum_{11}^{\lambda}(m, n ; x, y)\right\|_{r}\right)=0 .
$$

Thus, $\left\|S_{m n}-V_{m n}^{\lambda}\right\|_{r} \rightarrow 0$ as $\min \{m, n\} \rightarrow \infty$.

Acknowledgements. The authors are very thankful to the referee for his/her valuable suggestions.

\section{REFERENCES}

[1] C. P. Chen and Y. W. Chauang, $L^{1}$-convergence of double Fourier series, Chin. J. Math. 19(4) (1991), 391-410.

[2] K. Kaur, S. S. Bhatia and B. Ram, $L^{1}$-Convergence of complex double trigonometric series, Proc. Indian Acad. Sci. 113(3) (2003), 1-9.

[3] A. N. Kolmogorov, Sur l'ordre de grandeur des coefficients de la sèrie de Fourier-Lebesque, Bulletin L'Academie Polonaise des Science (1923), 83-86.

[4] F. Móricz, Convergence and integrability of double trigonometric series with coefficients of bounded variation, Proc. Amer. Math. Soc. 102 (1988), 633-640.

[5] F. Móricz, On the integrability and $L^{1}$-convergence of double trigonometric series, Studia Math. (1991), 203-225.

[6] T. M. Vukolova, Certain properties of trigonometric series with monotone coefficients (English, Russian original), Moscow Univ. Math. Bull. 39(6) (1984), 24-30, translation from Vestnik Moskov. Univ. 1(6) (1984), 18-23.

[7] W. H. Young, On the Fourier series of bounded functions, Proc. London Math. Soc. 12(2) (1913), 41-70.

[8] A. Zygmund, Trigonometric Series, Vols. I, II, Cambridge University Press, Cambridge, 1959. 
${ }^{1}$ Department of Applied Mathematics,

GZS Campus College of Engineering and Technology,

Maharaja Ranjit Singh Punjab Technical University Bathinda, Punjab, India

Email address: karanvir@mrsptu.ac.in

${ }^{2}$ Department of Mathematics,

Amity University of RAJASTHAN,

JAIPUR, INDIA

Email address: kmodi@jpr.amity.edu 